\title{
Design de Serviço para Inovação Social: uma revisão bibliométrica da literatura.
}

Service Design for Social Innovation: a bibliometric review of the literature.

\author{
BERLATO, Larissa Fontoura; Mestranda; Universidade Federal de Santa Catarina \\ lari.berlato@gmail.com \\ FERRARI, Aldo Sigolo; aldoferrari@uol.com.br \\ FIGUEIREDO, Luiz Fernando Gonçalves de; Doutor; Universidade Federal de Santa Catarina \\ Iffigueiredo2009@gmail.com
}

GOMES FERREIRA, Marcelo Gitirana; Doutor; Universidade Federal de Santa Catarina marcelo.gitirana@gmail.com

\begin{abstract}
Resumo
Diante da necessidade de soluções para os complexos problemas sociais e ambientais enfrentados atualmente, a inovação social é uma alternativa para o desenvolvimento de soluções sustentáveis, alinhando o desenvolvimento local ao crescimento econômico, a fim de proporcionar uma mudança social e melhores oportunidades aos cidadãos (ANDRÉ E ABREU, 2006; BIGNETTI, 2011; CAJAIBASANTANA, 2014). O Design de Serviço tem potencial para ampliar a inovação social, identificando as demandas locais e desenvolvendo soluções inovadoras considerando os valores locais, de forma colaborativa. O objetivo deste estudo é identificar, através de uma revisão bibliométrica sistematizada, as pesquisas científicas recentes presentes nas principais bases de dados que tenham relação com Design de Serviço para inovação social. Como resultado foram encontrados 42 artigos que passaram por um processo de filtragem em três diferentes etapas resultando em um portfólio bibliográfico de 13 artigos relevantes para pesquisas na área de Design de Serviço para a inovação social.
\end{abstract}

Palavras Chave: Design; Design de Serviço; Inovação Social e revisão bibliométrica.

\begin{abstract}
Given the need for solutions to the complex social and environmental problems currently faced, social innovation is an alternative for the development of sustainable solutions, aligning local development with economic growth, in order to provide social change and better opportunities for citizens (ANDRÉ E ABREU, 2006; BIGNETTI, 2011; CAJAIBA-SANTANA, 2014). Service Design has the potential to broaden social innovation by identifying local demands and developing innovative solutions that take local values into account in a collaborative way. The aim of this study is to identify, through a systematized bibliometric review, the recent scientific research present in the main databases that have a relation with Service Design for social innovation. As a result, 42 articles were found that underwent a filtering process in three different stages resulting in a bibliographic portfolio of 13 articles relevant to research in the area of Service Design for social innovation.
\end{abstract}

Keywords: Design; Service Design; Social Innovation and bibliometric review. 


\section{Introdução}

Os modelos de produção e consumo vigentes desde a Revolução Industrial, bem como os valores que os sustentam, estão em desacordo com as bases necessárias para a manutenção de um ambiente sadio, pois vêm comprometendo o futuro do nosso planeta (BRUNDTLAND, 1987). A transição para a sustentabilidade requer uma transformação na maneira como produzimos, consumimos e no modo como vivemos (CASTELLS, 1999, MANZINI, 2008, UNDSD, 1992). Neste contexto, surge uma economia social, que avança de forma veloz, fazendo com que novos programas e conceitos surjam com foco na eliminação dos problemas de desigualdade social, mudanças climáticas, poluição ambiental, doenças crônicas e injustiças sociais (BIGNETTI, 2011). Esse novo pensar deve alinhar o desenvolvimento local ao crescimento econômico, a fim de proporcionar uma mudança social e melhores oportunidades aos cidadãos (ANDRÉ E ABREU, 2006; BIGNETTI, 2011; CAJAIBA-SANTANA, 2014). A inovação social é uma nova solução para um problema social, mais eficaz, eficiente, sustentável do que as soluções atuais e para os quais o valor criado acumula principalmente para a sociedade como um todo, em vez de indivíduos privados (CENTER FOR SOCIAL INNOVATION - STANFORD UNIVERSITY, 2015).

Com a mudança da economia de manufatura para a provisão de informações e serviços, a economia de serviços tem sido responsável por $60-70 \%$ do produto interno bruto dos países desenvolvidos (MAGER, 2009). Neste contexto de aumento da importância dos fluxos de serviço surge o Design de Serviço, área de estudo dedicada a desenvolver soluções para o setor de serviços. O Design de Serviço aborda a funcionalidade e a forma de prestação dos serviços a partir da perspectiva do usuário. Seu objetivo é desenvolver as interfaces do serviço de modo que essas sejam úteis, utilizáveis, e desejáveis do ponto de vista do usuário e eficazes, eficientes e distintas do ponto de vista do prestador do serviço (MAGER, 2009). O Design de Serviço tem sido considerado um catalisador da inovação na política nacional, desenvolvimento regional e inovação empresarial (EUROPEAN COMISSION, 2011). A alternativa de conceber serviços que criam não só benefícios econômicos, mas também valor social, promovem uma mudança social em seus contextos (MANZINI, 2014; JOLY, STRAIOTO E FIGUEIREDO, 2015). Esta pesquisa se propõe a identificar, através de uma revisão bibliométrica sistematizada, as pesquisas científicas recentes presentes nas principais bases de dados que tenham relação com Design de Serviço para inovação social.

\section{Contextualização Teórica}

\subsection{Inovação Social}

No Manual de Oslo, uma das primeiras tentativas de orientar e padronizar a inovação de países industrializados, inovação se refere à "introdução de um bem ou serviço novo ou significativamente melhorado no que concerne à suas características ou usos previstos" (OCDE, 2005 , p. 57). A inovação é o motor do crescimento empresarial, social e econômico do mundo. Através do processo de inovação surgem novos produtos, processos e serviços, além de novas experiências e alternativas para resolução dos problemas advindos do mundo contemporâneo e globalizado, em que estamos inseridos (TIDD E BESSANT, 2015).

Paralelo a economia capitalista, surge uma economia social, que avança de forma veloz, fazendo com que novos programas e conceitos surjam com foco na eliminação dos problemas de desigualdade social, mudanças climáticas, poluição ambiental, doenças crônicas e injustiças sociais (BIGNETTI, 2011). Neste contexto, a inovação, inicialmente vinculada a geração de lucros e ganhos econômicos nos negócios, amplia seu foco como uma alternativa para o desenvolvimento de 
soluções sustentáveis. A inovação com vistas a uma mudança social, não exclui a criação e a geração de valor, entretanto, diferente do valor comercial, o valor gerado na inovação social é voltado para a sociedade, criando um "impacto social positivo", reconhecido como "valor social" (MULGAN, 2006; BIGNETTI, 2011). De forma geral, inovação social é uma nova solução para um problema social que é mais eficaz, eficiente, sustentável, do que as soluções atuais e para os quais o valor criado acumula principalmente para a sociedade como um todo, em vez de indivíduos privados (CENTER FOR SOCIAL INNOVATION - STANFORD UNIVERSITY, 2015).

Inovação social é um processo de mudança que emerge da recombinação criativa de elementos existentes (de capital social à herança cultural, do artesanato tradicional ao acesso de tecnologia avançada), que busca alcançar objetivos socialmente reconhecidos de uma nova forma (MANZINI, 2014). Para o autor, o processo de mudança está ligado à inovação, cuja base parte de uma nova recombinação de elementos já existentes em um dado meio, capazes de criar benefícios sociais reconhecidos.

As inovações sociais são entendidas como soluções sociais alternativas, baseadas em atores e suas inter-relações como recursos sociais, que encontram novas maneiras de atender às demandas sociais e/ou gerar mudanças sociais no nível sistêmico (promovendo mudanças incrementais e radicais nos sistemas de produção e consumo, por exemplo). (CAJAIBASANTANA, 2014, p. 44).

As inovações sociais são motivadas por mudanças de comportamento (mais do que por tecnologia ou mercado) e elas emergem de processos bottom-up (mais do que de processos topdown) (MANZINI, 2008). O processo bottom-up, quando a iniciativa surge pela auto-organização de atores sociais, é visto como o motor das inovações sociais, onde o papel dos cidadãos é de codesenvolvedor ativo, resultando no empoderamento dos mesmos (RUBALCABA et al., 2013).

\subsection{Design de serviço}

O Design de Serviço é considerado um campo de pesquisa ainda novo, surgido no início de 1990, e sob pressão das mudanças no mercado tem se desenvolvido rapidamente (MAGER, 2006). O Design de Serviço é o pensamento de Design aplicado ao desenvolvimento de serviços para as pessoas e com as pessoas, permitindo a colaboração e a cocriação. Esta abordagem do Design, torna o serviço útil, utilizável, desejável para os clientes e eficiente e eficazes para as organizações (MORITZ, 2005) onde todos os pontos de contato são projetados visando a melhor experiência do usuário (SERVICE DESIGN NETWORK - SDN, 2016). Esta área estuda as interações entre todos os stakeholders envolvidos no serviço, e não apenas o usuário (STICKDORN E SCHNEIDER, 2010), é uma abordagem que ajuda o provedor a antecipar a experiência do usuário, entender suas necessidades e expectativas e desenvolver conjuntamente uma oferta junto com os usuários (COOK et al., 2002).

O Design voltado para o desenvolvimento de serviços é uma abordagem interdisciplinar que combina diferentes métodos e ferramentas de diversas disciplinas e se apoia em cinco princípios básicos que contribuem com que o processo de desenvolvimento do produto tangencie a inovação: centrado no usuário, cocriativo, sequencial, evidente e holístico (STICKDORN E SCHNEIDER, 2010).

O Fórum LEED da OCDE sobre Inovação Social (OCDE, 2000) e a Comissão Européia (2011) destacaram o vínculo entre serviços e inovação social. Os serviços são baseados nas relações entre atores (VARGO E LUSCH, 2011), nesse sentido, os serviços podem melhorar suas capacidades inovadoras ao pensar em novas formas de interações sociais, parcerias e cocriação de valor (CIPOLLA e MANZINI, 2009) se tornando um motor para transformações societárias mais amplas (SANGIORGI, 2011). Os setores de serviços mais notáveis em que as inovações sociais ocorrem são 
a educação (em escolas), saúde (em modelos de cuidados de saúde centrados no paciente), turismo (em iniciativas de desenvolvimento rural), finanças (em crowdfunding, microfinanças) e serviços sociais (em empreendedorismo social) (WINDRUM et al, 2016).

\section{Procedimentos Metodológicos}

A revisão da literatura é um processo de levantamento e análise do que já foi publicado sobre o tema e o problema de pesquisa escolhidos (SILVA e MENEZES, 2005). A bibliometria é definida como uma ferramenta estatística para avaliar e medir dados por meio de mapeamento e geração de indicadores de tratamento e gestão da informação e do conhecimento (HAYASHY e LETA, 2013). Com o objetivo de identificar as pesquisas realizadas na área de Design de Serviço visando a inovação social realizou-se uma revisão sistemática da literatura baseada na bibliometria. Para a seleção e avaliação de estudos foram estabelecidos os seguintes critérios:

\section{A. Critérios para Inclusão (requisitos simultâneos):}

- ESCOPO: Pesquisas que abordem a contribuição do Design de Serviço para a inovação social.

- TIPO DE REFERÊNCIA: Artigos de periódicos publicados ou aceitos para publicação.

- ACESSO: Trabalhos acessíveis através do: (1) Portal de Periódicos da CAPES na UFSC; (2) Google Acadêmico; (3) portal das editoras, de forma gratuita; (4) através do sistema COMUT nas instituições envolvidas.

- IDIOMA: Escritos em idiomas dominados pela autora: Português, Inglês e Espanhol.

- ANO: Trabalhos recentes, publicados nos últimos 5 anos.

B. Critérios para Exclusão (requisitos simultâneos):

- ESCOPO: Pesquisas que abordem Design de Serviço sem foco na inovação social e pesquisas que abordem inovação social sem a contribuição do Design de Serviço.

- TIPO DE REFERÊNCIA: Artigos de anais de congresso ou entrevistas publicadas em periódicos.

- $\quad$ ACESSO: Trabalhos, cuja obtenção envolva o pagamento direto por parte dos participantes da pesquisa (excetuando aqueles obtidos pelo sistema COMUT) e trabalhos obtidos de forma cuja legalidade possa ser questionada (Sci-Hub, por exemplo).

- IDIOMA: Trabalhos escritos em idiomas não dominados pelos participantes da pesquisa.

Durante o processo de seleção, as referências encontradas na Scopus, na Web of Science e na Scielo, foram exportadas - com todas as suas informações, incluindo as referências citadas - no formato .bibtex para, em seguida, importadas no gerenciador de referências Mendeley. A partir de então aplicaram-se as seguintes filtragens consecutivas conforme a Tabela 1:

Tabela 1 - Filtragens da RSL

\begin{tabular}{|c|c|c|c|}
\hline FILTRO 1 & FILTRO 2 & FILTRO 3 \\
\hline $\begin{array}{c}\text { Através do gerenciador de } \\
\text { referências Mendeley verificou-se a } \\
\text { existência de referências duplicadas, } \\
\text { as quais foram excluídas da seleção. }\end{array}$ & $\begin{array}{c}\text { Leitura de Título, Resumo e Palavras- } \\
\text { chave, excluindo as publicações de } \\
\text { acordo com os critérios de exclusão } \\
\text { estabelecidos anteriormente. }\end{array}$ & $\begin{array}{c}\text { Leitura completa dos artigos, excluindo as publicações de acordo } \\
\text { cos critérios de inclusão e exclusão. Para esta filtragem, foram } \\
\text { buscados os pdfs das referências selecionadas na segunda }\end{array}$ \\
$\begin{array}{c}\text { filtragem. As referências para as quais não for possível a obtenção } \\
\text { dos arquivos .pdf pelos meios descrito nos critérios para inclusão } \\
\text { e/ou referências que se enquadrem nos itens de critérios para } \\
\text { exclusão foram excluídas do portfólio de referências analisado. }\end{array}$
\end{tabular}

Fonte: Elaborado pela autora 


\section{Artigo Completo}

\section{Revisão Bibliométrica da Literatura}

As buscas foram realizadas nas bases de dados, no dia 20 de outubro de 2017. Visando expandir os resultados da busca, foram definidas as seguintes palavras-chave com a aplicação da língua inglesa:

- $\quad$ Service Design, Service Development, Service Innovation

- $\quad$ Social Innovation, Social Entrepreneurship, Social Change

Com a definição das palavras-chave construiu-se à seguinte string de busca: ("service design" OR "service development" OR "service innovation") AND ("social innovation" OR "social entrepreneurship" OR "social change").

O critério de seleção das fontes de busca foi as maiores bases de dados na área de Ciências Sociais que incluam artigos em periódicos que possam ser acessadas através do sistema CAPES, resultando na seguinte lista de fontes de busca: Elsevier Scopus, Web of Science e Scielo. Foram buscados somente os artigos de periódicos publicados ou aceitos para publicação nos últimos 5 anos. Com relação ao acesso foram analisados os trabalhos acessíveis através do Portal de Periódicos da CAPES na UFSC; Google Acadêmico; portal das editoras, de forma gratuita e através do sistema COMUT nas instituições envolvidas. Quanto à língua, foram buscados apenas aquelas referências escritas nos idiomas dominados pela autora: português, inglês e espanhol. Com relação a área de pesquisa, nas bases Elsevier Scopus e Web of Science não foram aplicados filtro de área visando ampliar a pesquisa. Na base Scielo foi aplicado filtro de área por Ciências Sociais. O resultado encontrado foi de 42 publicações conforme a Tabela 2:

Tabela 2 - Resultados da Busca

\begin{tabular}{|c|c|c|c|c|}
\hline ALGORITMO DE ARGUMENTO DE BUSCA & SCIELO & $\begin{array}{l}\text { WEB OF } \\
\text { SCIENCE }\end{array}$ & SCOPUS & TOTAL \\
\hline $\begin{array}{l}\text { ("service design" OR "service } \\
\text { development" OR "service innovation") } \\
\text { AND ("social innovation" OR "social } \\
\text { entrepreneurship" OR "social change") }\end{array}$ & 10 & 10 & 22 & 42 \\
\hline
\end{tabular}

Fonte: Elaborado pela autora com base na pesquisa realizada em 20 out 2017.

O primeiro processo de filtragem foi a identificação das publicações repetidas por meio do uso do gerenciador bibliográfico Mendeley. Nesta etapa foram excluídos por títulos as publicações repetidas, resultando em 33 artigos. O segundo processo de filtragem se refere à leitura dos títulos, resumo e palavras-chave dos artigos com o objetivo de verificar a adequação destes com o tema da pesquisa, permanecendo 20 artigos que demonstraram relevância com o tema. Posteriormente, foi realizada um terceiro filtro a partir da leitura integral dos artigos, permanecendo 13 artigos que estão de acordo com os critérios de inclusão e exclusão estabelecidos nesta RSL. Os resultados de cada etapa podem ser verificados na Figura 1: 
Figura 1 - Resultado do Portfolio de Referências

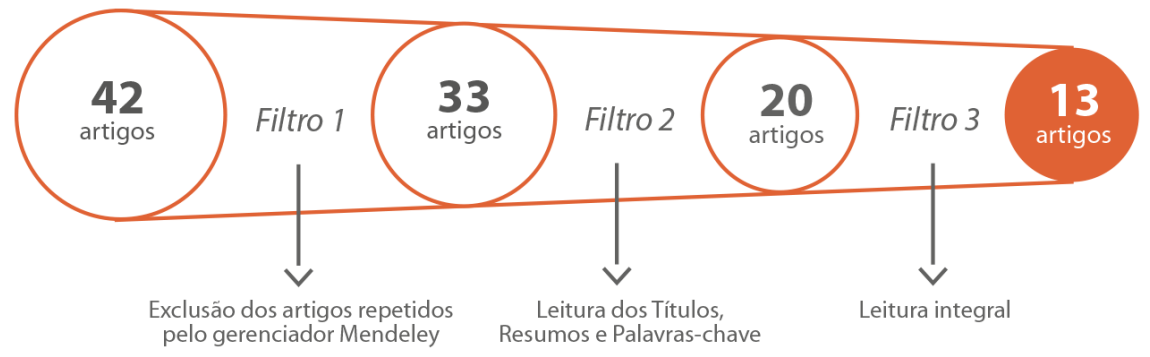

Fonte: Elaborado pela autora.

Visando favorecer a sistematização e a visualização do processo desta RSL foi desenvolvido um diagrama de bloco conforme a Figura 2:

Figura 2 - Diagrama de bloco da RSL sobre a contribuição do Design de Serviço para a inovação social.

(A) (B)

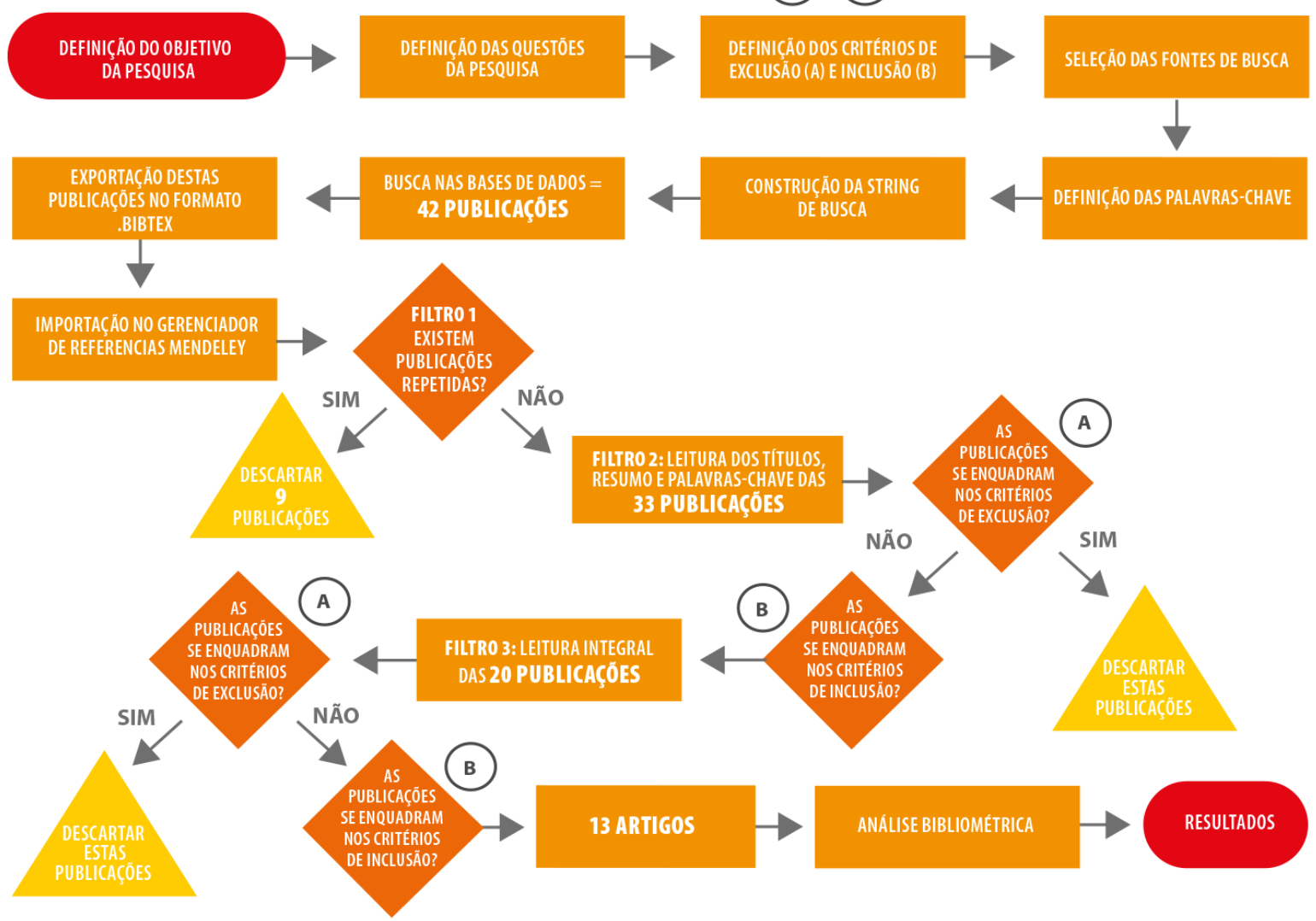

Fonte: Elaborado pela autora.

Por fim, obteve-se um Portfólio Final de Referências contendo 13 artigos conforme a Tabela 3: 


\section{Artigo Completo}

Tabela 3 - Portfolio Final de Referências

Ewert, B., \& Evers, A. (2014). Blueprints for the future of welfare provision? shared

1 features of service innovations across europe. Social Policy and Society, 13(3), 423-

SCOPUS

ARTIGO 432. doi:10.1017/S1474746414000074

Fassi, D., \& Motter, R. (2017). System-events toolbox: Designing a system of events

2 that relies on local resources to create urban places for social cohesion

SCOPUS

ARTIGO doi:10.1007/978-3-319-57070-9_19

Galli, F., Brunori, G., Di lacovo, F., \& Innocenti, S. (2014). Co-producing sustainability:

Involving parents and civil society in the governance of school meal services. A case study from Pisa, Italy. Sustainability (Switzerland), 6(4), 1643-1666. doi:10.3390/su6041643

$4 \quad$ Hyytinen, K., \& Toivonen, M. (2015). Future energy services: Empowering local communities and citizens. Foresight, 17(4), 349-364. doi:10.1108/FS-08-2013-0035 SCOPUS ARTIGO Kidd, S., \& McKenzie, K. (2014). Social entrepreneurship and services for marginalized

5 groups. Ethnicity and Inequalities in Health and Social Care, 7(1), 3-13.

SCOPUS ARTIGO doi:10.1108/EIHSC-03-2013-0004

6 Mariñez-Navarro, F. (2016). Conhecimento cívico na inovação da gestão pública. Convergência , 23 (70), 87-114.

SCIELO

Minemoto, T., Miyamoto, A., Doi, M., Bando, A., Hirasawa, S., Sano, Y., \& Matsumura,

7 S. (2016). Application of service design and vision design by collaborative creation in SCOPUS ARTIGO urban development business. Hitachi Review, 65(2), 853-859.

8 Muhamad, N. S., \& Adham, K. A. (2013). Social entrepreneurship as transformative service for societal well-being. Jurnal Pengurusan, 39

Petersen, S. A., Concilio, G., \& Oliveira, M. (2015). Smart neighbourhood learning - the case of myneighbourhood. Interaction Design and Architecture(s), 27(1), 66-78. Ret

Srivastava, S. C., \& Shainesh, G. (2015). Bridging the Service Divide Through Digitally

10 Enabled Service Innovations: Evidence from Indian Healthcare Service Providers. Mis Quarterly, 39(1).

Vickers, I., Lyon, F., Sepulveda, L., \& McMullin, C. (2017). Public service innovation and multiple institutional logics: The case of hybrid social enterprise providers of health and wellbeing. Research Policy, 46(10), 1755-1768.

ARTIGO doi:10.1016/j.respol.2017.08.003

Windrum, P., Schartinger, D., Rubalcaba, L., Gallouj, F., \& Toivonen, M. (2016). The cocreation of multi-agent social innovations: A bridge between service and social innovation research. European Journal of Innovation Management, 19(2), 150-166. doi:10.1108/EJIM-05-2015-0033

Yang, C. \& Sung, T. (2016). Service design for social innovation through participatory action research. International Journal of Design, 10(1), 21-36.

Fonte: Elaborado pela autora.

\subsection{Análises Bibliométricas do Portfolio Final de Referências}

A seguir, apresentam-se as análises bibliométricas, aplicando métodos estatísticos e matemáticos para analisar e construir indicadores sobre a dinâmica e a evolução das pesquisas que vêm sendo (ou já foram) realizadas sobre a contribuição do Design de Serviço para a inovação social conforme a Tabela 4: 


\section{Artigo Completo}

Tabela 4 - Portfolio Final de Referências relativo ao ano, país, afiliação, área e fonte.

\begin{tabular}{|c|c|c|c|c|c|}
\hline REF. & ANO & PAÍs & AFILIAÇÃO & ÁREA & FONTE \\
\hline 1 & 2014 & $\begin{array}{l}\text { REINO } \\
\text { UNIDO }\end{array}$ & $\begin{array}{l}\text { Justus Liebig University } \\
\text { Giessen }\end{array}$ & Social Sciences & $\begin{array}{l}\text { Social Policy \& } \\
\text { Society }\end{array}$ \\
\hline 2 & 2017 & ITÁLIA & Politecnico di Milano & $\begin{array}{l}\text { Engineering; Energy e } \\
\text { Environmental Science }\end{array}$ & $\begin{array}{l}\text { Green Energy and } \\
\text { Technology }\end{array}$ \\
\hline 3 & 2014 & ITÁLIA & $\begin{array}{l}\text { Laboratorio di Studi Rurali } \\
\text { Sismondi; Universita di Pisa }\end{array}$ & $\begin{array}{l}\text { Social Sciences; Energy and } \\
\text { Environmental Science }\end{array}$ & Sustainability \\
\hline 4 & 2015 & FINLÂNDIA & $\begin{array}{l}\text { VTT Technical Research } \\
\text { Centre of Finland }\end{array}$ & $\begin{array}{c}\text { Decision Sciences Economics } \\
\text { and Econometrics and } \\
\text { Finance }\end{array}$ & Foresight \\
\hline 5 & 2014 & CANADÁ & University of Toronto & $\begin{array}{l}\text { Social Sciences; } \\
\text { Medicine e Psychology }\end{array}$ & $\begin{array}{l}\text { Ethnicity and } \\
\text { Inequalities in Health } \\
\text { and Social Care }\end{array}$ \\
\hline 6 & 2016 & MÉXICO & Tecnologico de Monterrey & Social Sciences & Convergência \\
\hline 7 & 2016 & JAPÃO & Hitachi, Ltd. & $\begin{array}{l}\text { Engineering; Computer } \\
\text { Science; Energy and } \\
\text { Chemical Engineering }\end{array}$ & Hitachi Review \\
\hline 8 & 2013 & MALÁSIA & $\begin{array}{c}\text { Universiti Kebangsaan } \\
\text { Malaysia }\end{array}$ & $\begin{array}{l}\text { Business, Management and } \\
\text { Accounting }\end{array}$ & Jurnal Pengurusan \\
\hline 9 & 2015 & $\begin{array}{l}\text { NORUEGA } \\
\text { ITÁLIA }\end{array}$ & $\begin{array}{l}\text { Politecnico di Milano; } \\
\text { Norwegian Institute of } \\
\text { Technolog }\end{array}$ & $\begin{array}{l}\text { Engineering and Computer } \\
\text { Science }\end{array}$ & $\begin{array}{l}\text { Interaction Design } \\
\text { and Architecture(s) } \\
\text { Journal }\end{array}$ \\
\hline 10 & 2015 & EUA & $\begin{array}{l}\text { HEC School of Management; } \\
\text { Indian Institute of } \\
\text { Management Bangalore }\end{array}$ & $\begin{array}{c}\text { Business, Management and } \\
\text { Accounting; Computer } \\
\text { Science and Decision } \\
\text { Sciences }\end{array}$ & $\begin{array}{c}\text { MIS Quarterly: } \\
\text { Management } \\
\text { Information Systems }\end{array}$ \\
\hline 11 & 2017 & $\begin{array}{l}\text { REINO } \\
\text { UNIDO }\end{array}$ & $\begin{array}{l}\text { Middlesex University } \\
\text { University of Birmingham }\end{array}$ & $\begin{array}{c}\text { Business, Management and } \\
\text { Accounting } \\
\text { Decision Sciences }\end{array}$ & Research Policy \\
\hline 12 & 2016 & $\begin{array}{c}\text { REINO } \\
\text { UNIDO } \\
\text { AUSTRIA } \\
\text { ESPANHA } \\
\text { FRANÇA E } \\
\text { FINLÂNDIA }\end{array}$ & $\begin{array}{c}\text { VTT Technical Research } \\
\text { Centre of Finland; Universite } \\
\text { des Sciences et Technologies } \\
\text { de Lille; University of } \\
\text { Nottingham; } \\
\text { Universidad de Alcala; } \\
\text { Austrian Institute of } \\
\text { Technology }\end{array}$ & $\begin{array}{l}\text { Business, Management and } \\
\text { Accounting }\end{array}$ & $\begin{array}{l}\text { European Journal of } \\
\text { Innovation } \\
\text { Management }\end{array}$ \\
\hline 13 & 2016 & TAIWAN & $\begin{array}{l}\text { National Taiwan University of } \\
\text { Science and Technology }\end{array}$ & $\begin{array}{c}\text { Business, Management and } \\
\text { Accounting } \\
\text { Computer Science }\end{array}$ & $\begin{array}{l}\text { International Journal } \\
\text { of Design }\end{array}$ \\
\hline
\end{tabular}

Fonte: Elaborado pela autora.

Com relação à distribuição dos artigos nos anos de publicação, percebe-se um aumento do número e da frequência nos últimos anos conforme a Figura 3. Em 2013 houve a ocorrência de apenas 1 artigo, em 2014 e 2015 houve a constância de 3 artigos por ano e o ápice se deu em 2016 com 4 artigos publicados. É possível que este aumento no número de publicações seja mantido visto que até a presente pesquisa já constavam 2 artigos publicados em 2017. Verifica-se que não existe um periódico mais relevante para o tema nos últimos 5 anos, conforme a Figura 4: 
Figura 3 - Evolução das publicações ao longo dos anos

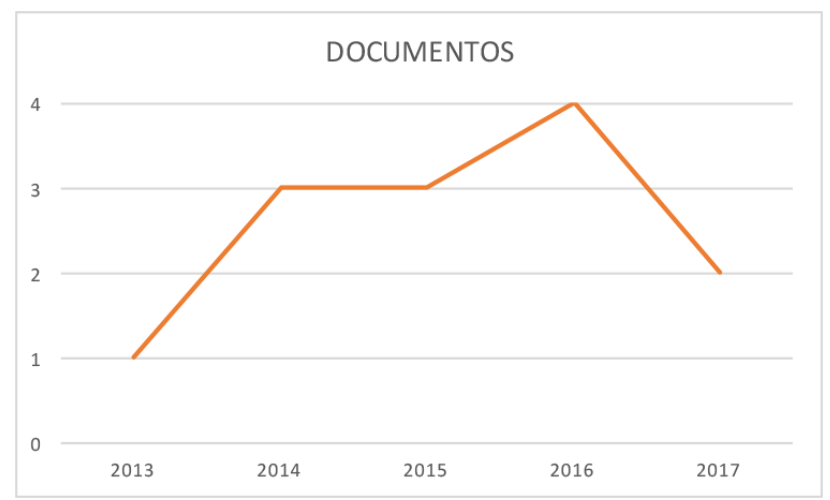

Fonte: Scopus, 2017.

Figura 4-Documentos por fonte.

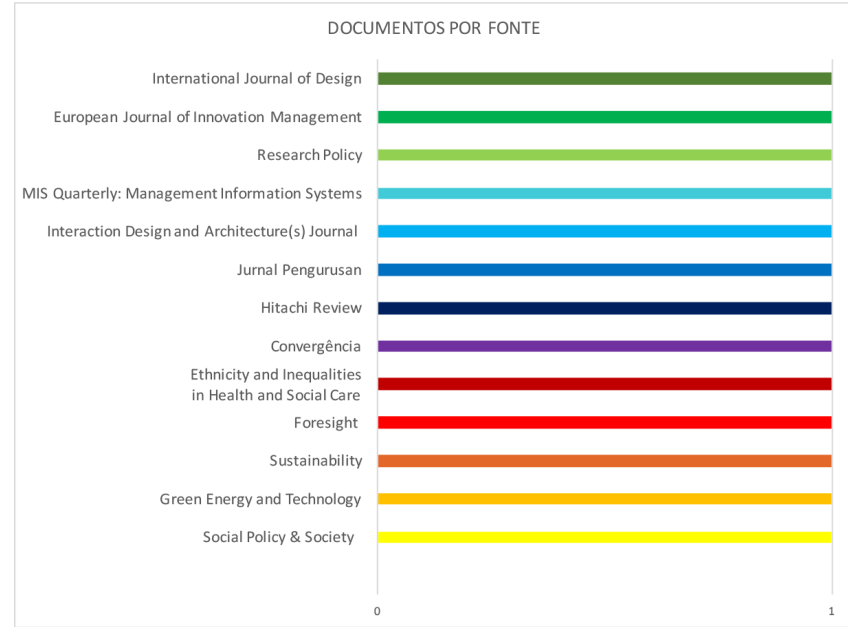

Fonte: Scopus, 2017.

O índice SCImago Journal Rank (SJR) avalia os periódicos científicos a partir da quantidade de citações por eles recebidas nos 3 anos que se seguem à publicação das edições, levando em consideração a origem das citações (por qualidade dos periódicos) e também a lógica de citações própria a cada subárea. Visando um caráter mais qualitativo e contextual optou-se por avaliar as principais fontes deste Portfólio Bibliográfico através do índice SJR conforme Figura 5:

Figura 5 - Índice SCImago Journal Rank (SJR) por ano

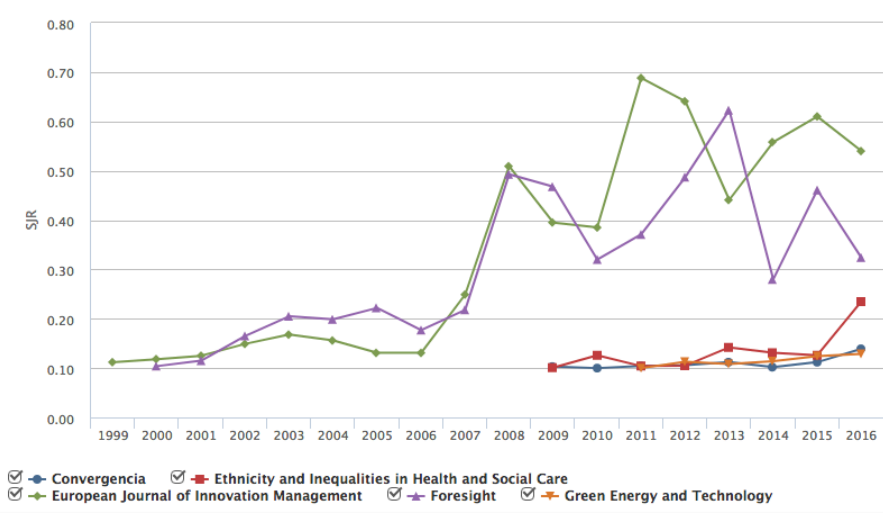


Fonte: Scopus, 2017.

Com relação aos países que mais tem publicado nos últimos 5 anos, pode-se identificar a Itália ( 3 artigos) e Reino Unido ( 3 artigos) como os países que mais tem publicado artigos relacionados a este tema, conforme Figura 6 . No que tange às instituições com o maior número de publicações identifica-se o Politécnico de Milão, na Itália, país com o maior número de publicações e o VTT Technical Research Centre of Finland, na Finlândia, país com o segundo maior número de publicações, conforme a Figura 7:

Figura 6 - Documentos por país

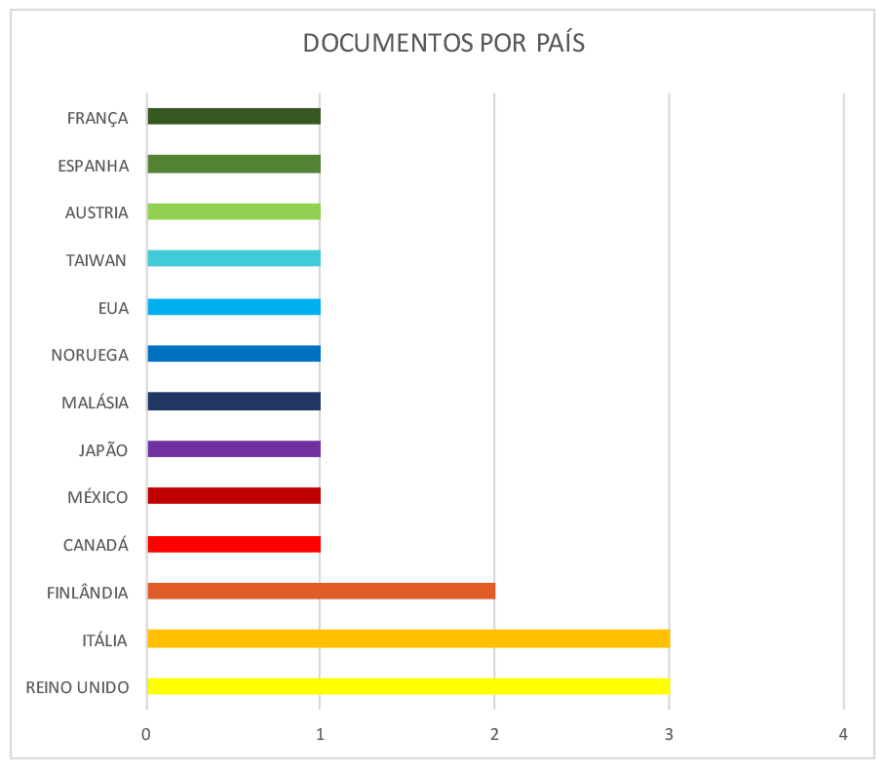

Fonte: Scopus, 2017.

Figura 7- Documentos por instituição

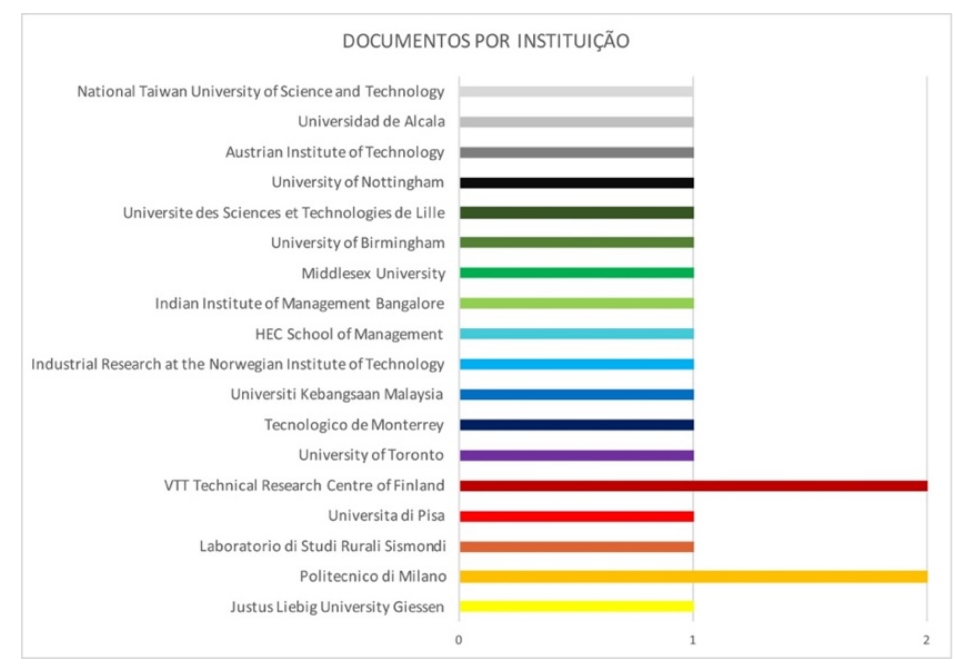

Fonte: Scopus, 2017.

Com relação à autoria das publicações não se identificou nenhum autor mais relevante para o tema nos últimos 5 anos, conforme a Figura 8: 
Figura 8 - Documentos por autor (comparação incluindo até 15 autores)

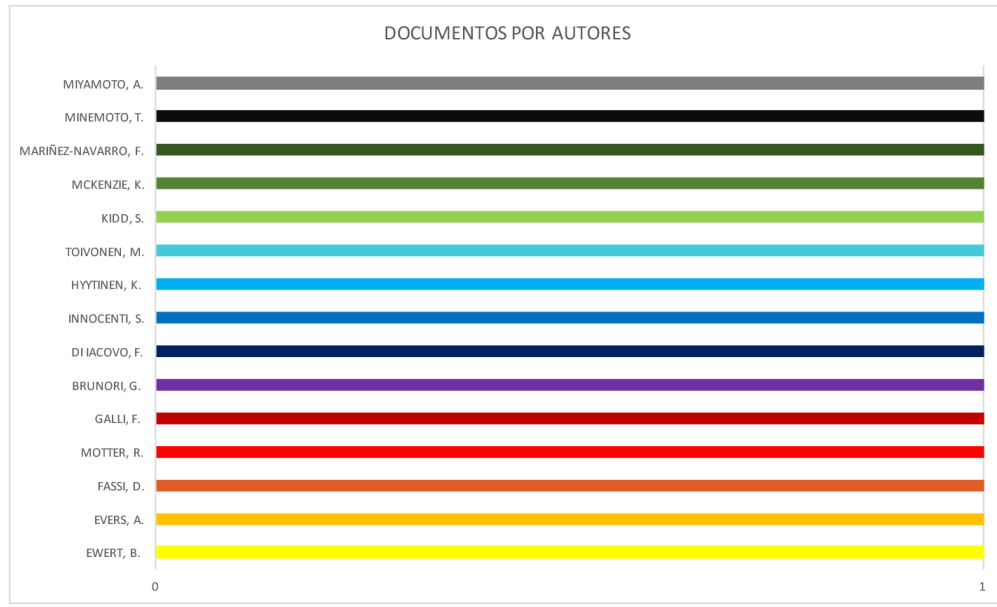

Fonte: Scopus, 2017.

As áreas de conhecimento com o maior número de ocorrências foram primeiramente Negócios, Gestão e Contabilidade, responsável por 18\% dos documentos; seguidos por Ciências Sociais e Ciências da Computação, ambas responsáveis por $14 \%$ dos documentos conforme a Figura 9:

Figura 9 - Documentos por área.

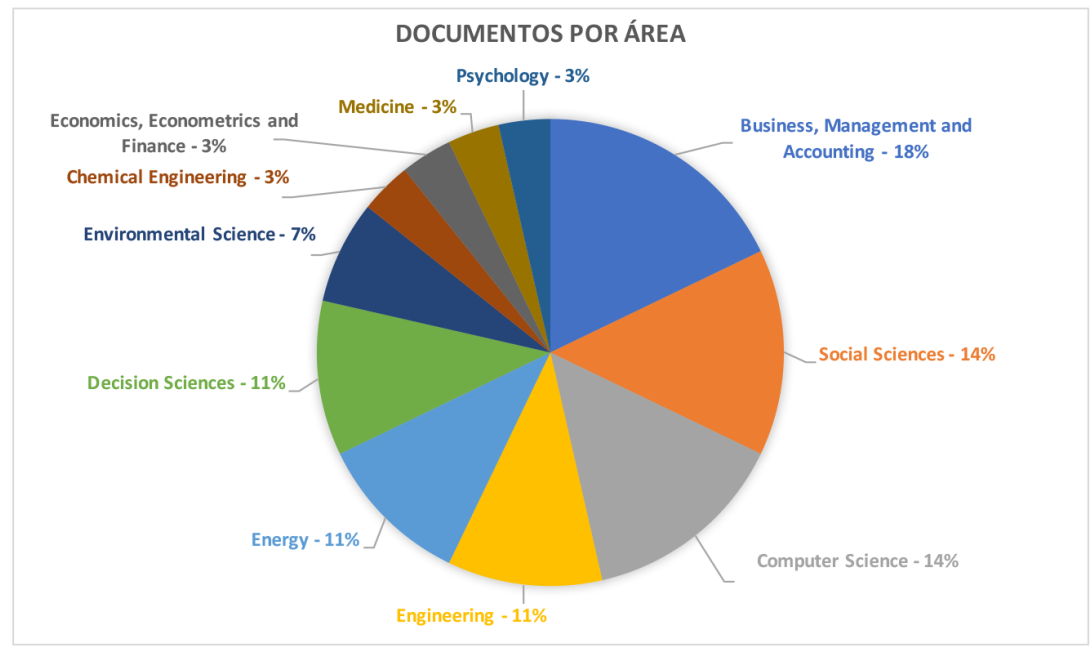

Fonte: Scopus, 2017.

A grande maioria das publicações são estudos de caso, principalmente oriundos da Europa. Ewert e Evers (2014) analisaram a inovação social no projeto europeu 'Welfare Innovations at the local Level in favour of Cohesion' (WILCO); Fassi e Motter (2017) apresentaram uma TOOLBOX de eventos para a coesão social utilizada no Politécnico de Milão, Itália; Galli et al (2014) analisaram uma parceria público-privada no serviço de refeições escolares em Pisa, Itália; Kidd e McKenzie (2014) examinaram a contribuição do Empreendedorismo Social em serviços de saúde mental em Toronto, Canadá; Minemoto et al (2016) apresentaram a aplicação de Design de serviço no projeto Kashiwa-no-ha Smart City, no Japão; Petersen, Concilio e Oliveira (2015) pesquisaram a perspectiva de cidades inteligentes a partir do projeto europeu MyNeighbourhood; Srivastava e Shainesh (2015) 


\section{Artigo Completo}

estudaram duas iniciativas de serviços na área da saúde, na Índia; Vickers et al (2017) realizaram um análise qualitativa em organizações de serviços de saúde e assistência social no Reino Unido; Windrum et al (2016) realizaram um estudo de caso, na Áustria, sobre a prestação de primeirossocorros em pacientes de problemas cardíacos; e Yang e Sung (2016) investigaram a plataforma de ação social 5\% Design Action, em Taiwan.

Algumas publicações são explorações teóricas sobre os temas que envolvem Design de Serviço e inovação social, como Hyytinen e Toivonen (2015) que sugerem inovações sociais em sistemas energéticos sustentáveis; Mariñez-Navarro (2016) que verificou a relação entre conhecimento cívico e inovação na gestão pública participativa; e Muhamad e Adham (2013) que analisaram o empreendedorismo social como um modelo de serviço transformador.

Um conjunto de redes foi desenvolvido com o auxílio do software VosViewer a partir do Portfólio Final de Referências da base de dados Scopus. Uma lista foi criada na Scopus, vinculada à conta pessoal da pesquisadora, com as 13 referências resultantes desta RSL. Em seguida, foi exportado um arquivo .csv com todos os dados de cada referência. Este arquivo foi então aberto no VOSviewer para a criação das redes que são apresentadas nas Figura 10 e Figura 11:

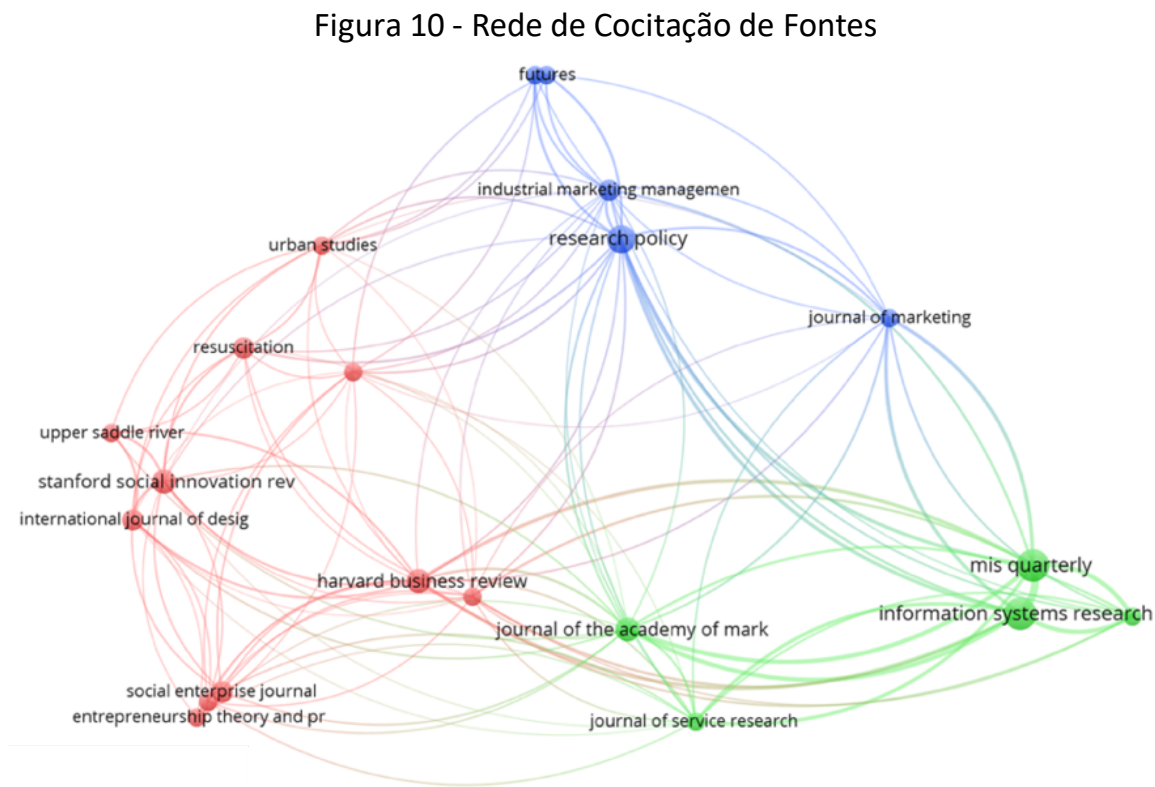

Fonte: VOSviwer, 2017

A cocitação é uma medida de correlacionamento (similaridade semântica) entre dois documentos (fontes ou autores). A rede de cocitação de fontes apresentada evidencia os principais periódicos mais cocitados (relacionados pelos temas que abordam) pelas 13 publicações do Portfólio Final de Referências. O tamanho dos círculos da rede (representativo de cada periódico) relaciona-se ao número de citações recebidas dentro do portfólio. Nesta rede, apenas os periódicos com três ( 3 ) ou mais citações foram representados (algo definido na construção da rede). A distância entre os círculos, por sua vez, se relaciona a proximidade temática dos periódicos. O vosviewer realiza a divisão dos periódicos por clusters temáticos. A partir desta rede, identificam-se 3 CLUSTERS de periódicos conforme Figura 10: 
1. VERMELHO: periódicos relacionados às áreas de Gestão de Negócios e Inovação Social, dentre os quais se destacam os periódicos Harvard Business Review e Stanford Social Innovation Review.

2. VERDE: periódicos relacionados a área de Gestão da Informação, dentre os quais se destacam os periódicos MIS Quarterly e Information Systems Research.

3. AZUL: periódicos relacionados a área de Inovação, Tecnologia e Marketing dentre os quais se destacam os periódicos Research Policy e Industrial Marketing Management.

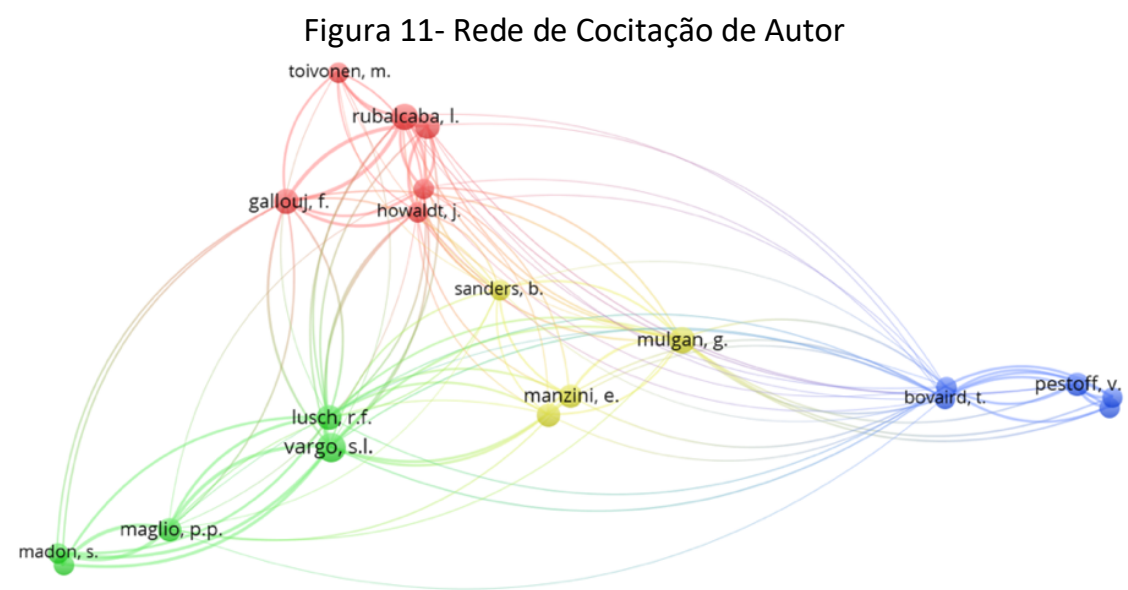

Fonte: VOSviwer, 2017.

A análise de cocitação de autores permite compreender a interlocução entre os pesquisadores inseridos na temática pesquisada. O tamanho dos círculos é proporcional à frequência de citação dos autores. Nesta rede, apenas os autores com quatro (4) ou mais citações foram representados (algo definido na construção da rede) conforme Figura 11. O VOSviewer realiza a divisão dos autores por clusters temáticos. Nesta rede, identificam-se 4 CLUSTERS:

1. VERMELHO: autores relacionados a área de Inovação de serviço, tendo como principal exponente Luis Rubalcaba.

2. VERDE: autores relacionados as áreas de Lógica Dominante de Serviço (S-D), tendo como principais exponentes Stephen Vargo e Robert Lusch.

3. AZUL: autores relacionados as áreas Gestão Estratégica do Setor Público, tendo como principal exponente Tony Bovaird e Victor Pestoff.

4. AMARELO: autores relacionados a área de Inovação social, tendo como principal exponente Ezio Manzini e Geoff Mulgan.

\section{Considerações Finais}

O propósito desta revisão bibliométrica da literatura foi o de identificar, a partir de uma criteriosa e objetiva seleção de pesquisas científicas, publicações, autores e grupos de pesquisa que apresentem conteúdos correlatos e fundamentais para somar um portfólio de referências que possa contribuir nas pesquisas na área de Design de Serviço para a inovação social. Do total de 42 artigos encontrados nesta revisão bibliométrica pode-se identificar 13 artigos que apresentam a contribuição do Design de Serviço visando uma inovação social. Todos os artigos resultantes foram analisados pelo ano de publicação, fonte, índice SJR, país, filiação, autor, área de conhecimento e rede de cocitação de fontes e de autor. No que tange aos resultados das análises bibliométricas, nos 
últimos 5 anos identifica-se um crescente interesse em publicações deste tema, possivelmente devido ao contexto atual de necessidade de mudanças sociais. A Europa se destaca nas publicações sobre este tema, sendo o Reino Unido e a Itália os países que mais tem publicado no período analisado. O Politécnico de Milão, na Itália, é um dos principais responsáveis pelas publicações relacionando Design de Serviço e inovação social e é a instituição de afiliação de Ezio Manzini, autor identificado na análise de rede de cocitação de autores e fundador e presidente de uma importante rede internacional de Design para a sustentabilidade, a Rede DESIS (Design for Social Innovation and Sustainability). As Ciências Sociais, área que abrange o Design, se configura como uma das áreas com maior número de publicações. A análise das redes de cocitação de fontes e de cocitação de autores permitiu expandir e aprofundar os conhecimentos sobre as fontes de publicação e sobre os autores mais relevantes em cada área que envolve os conceitos de Design de Serviço e inovação social.

A aplicação desse tema de estudo forneceu dados para pesquisas futuras, como também permitiu uma melhor percepção sobre o tema. A partir da análise do conteúdo dos artigos pode-se identificar uma crescente importância da inovação social no cenário atual bem como as contribuições do Design de Serviço no desenvolvimento de novas soluções para os problemas sociais por meio da integração de talentos e recursos no desenvolvimento de novas formas de interação e de parcerias, de forma colaborativa, visando a aprendizagem e o empoderamento dos cidadãos. Como oportunidade de pesquisa futura sugere-se um estudo sobre o desenvolvimento de Design de Serviço para a inovação social a partir de estudos de caso visando um melhor entendimento dos processos, técnicas, ferramentas e stakeholders envolvidos nos projetos de inovação social liderados pelo Design. Por fim, identifica-se a oportunidade de abordar essa temática por pesquisadores no Brasil, visto a falta de publicações nesta área em países em desenvolvimento.

\section{Referências}

ANDRÉ, I.; ABREU, A. Dimensões e Espaços da Inovação Social. Finisterra, v. XLI, n. 81, p. 121-141, 2006.

BIGNETTI, L. P. As inovações sociais: uma incursão por ideias, tendências e focos de pesquisa. Ciências Sociais Unisinos, v.47, n.1, p. 3-14, 2011.

BRUNDTLAND, G. et al. Our Common Future: The World Commission on Environment and Development. New York: ONU, 1987.

CAJAIBA-SANTANA, G. Social innovation: Moving the field forward. A conceptual framework. Technological Forecasting and Social Change, 2014. v. 82, p. 42-51, 2014.

CASTELLS, M. O poder da identidade. São Paulo: Paz e Terra, 1999.

CIPOLLA, C.; MANZINI, E. Relational services. Knowledge, Techn. \& Policy, v. 22, 2009. p. 45-50.

COOK, L.S. et al. Human issues in service design. Journal of Operations Management, v. 20, n. 1, pp. 159-174, 2002.

CSI-SU. In Center for Social Innovation - Stanford University, 2015. (http://csi.gsb.stanford.edu/)

EUROPEAN COMMISSION. In FP7 cooperation work programme 2011, Theme 8, socio-economic sciences and humanities, European Commission, Brussels, 2011. (http://ec.europa.eu/) 
HAYASHY, M. C. P. I.; LETA, J. Bibliometria e Cientometria: reflexões teóricas e interfaces. São Carlos: Pedro \& João Editores, 2013.

JOLY, M. P.; STRAIOTO, R.; FIGUEIREDO, L. F. Strategies in design for social innovation within Alto Vale Project. Strategic Research Design Journal. Porto Alegre: UNISINOS, 2015.

MAGER, B. Service design basics. Cologne: Köln International School of Design, 2006.

MAGER, B. Service Design as an Emerging Field. In: S. Miettinen; M. Kivisto; Designing Services with Innovative Methods. Helsink: Helsinki Univesity of Art and Design, 2009.

MANZINI, E. Design para a inovação social e sustentabilidade - Comunidades criativas, organizações colaborativas e novas redes projetuais. Rio de Janeiro: E-papers, 2008.

Making things happen: social innovation and design. Design Issues, v.30, n.1, p.57-66, 2014.

MORITZ, S. Service Design: pratical acess to an evolving field. Köln Cologne: International School of Design - University of Applied Sciences, 2005.

MULGAN, G. The Process of Social Innovation. Innovations, Governance, Globalization, v.1, n. 2 p. 145-162, 2006.

OECD. In Organização para a Cooperação e Desenvolvimento Econômico. LEED forum on social innovations, 2000. (http://www.oecd.org)

. Manual de Oslo: Diretrizes para coleta e interpretação de dados sobre inovação. ARTI/FINEP, 2005.

RUBALCABA, L.; DI MEGLIO, G.; GALLEGO, J. Public-private innovation networks and social innovation in the service economy. In Viñals, C.R. and Rodríguez, C.P.; Social Innovation: New Forms of Organization in Knowledge-Based Societies, Routledge, New York, NY, pp. 188-205, 2013.

SANGIORGI, D. Transformative services and transformation design. International Journal of Design, v. 5, n. 2, p. 29-40, 2011.

SDN. Sherlock, LEGO e o Service Design. 2016. (https://www.service-design-network.org)

SILVA, E. L. da; MENEZES, E. M. Metodologia da Pesquisa e Elaboração de Dissertação. $4^{\circ}$ edição. Florianópolis: UFSC, 2005.

STICKDORN, M.; SCHNEIDER, J. This is Service Design Thinking. Amsterdam: BiS Publishers, 2010.

TIDD, J.; BESSANT, J. Gestão da inovação. Porto Alegre: Bookman Editora, 2015.

UNDSD. In Agenda 21 - United Nations Conference on Environment \& Development. Rio de Janeiro: United Nations Division for Sustainable Development, 1992.

VARGO, S. L.; LUSCH, R. F. It's all b2b and beyond...: toward a systems perspective of the market. Industrial Marketing Management, v. 40, n. 2, p. 181-187, 2011.

WINDRUM, P. et al. The co-creation of multi-agent social innovations: A bridge between service and social innovation research. European Journal of Innovation Management, v.19, n.2, p.150-166, 2016. 